\section{HERZLICHEN GLÜCKWUNSCH!}

\section{Hans Kolaska feierte seinen 80. Geburtstag}

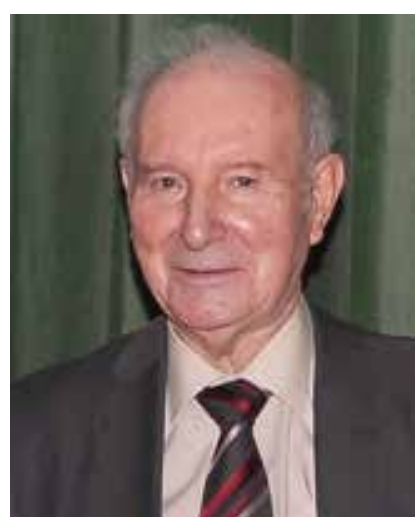

Was soll man über eine Persönlichkeit schreiben, die schon unzählige Ehrungen erhielt und die nicht nur auf dem Gebiet der Pulvermetallurgie von Hartmetallen, sondern weit darüber hinaus überaus geschätzt wird? Dass es sich hierbei um Hans Kolaska handelt, der am 29. September dieses Jahres seinen 80 . Geburtstag feierte, sollte sich - abgesehen von der Überschrift - bereits anhand des ersten Satzes erschließen.

Den Jubilar zeichnet eine Reihe von Eigenschaften aus, wie vor allem Zuverlässigkeit, Zielorientierung, Schaffensdrang, Hartnäckigkeit, Temperament, aber auch Empathie sowie eine ausgeprägte Verbindlichkeit. Ein Mann, ein Wort! Somit gestalten sich Absprachen mit ihm erfreulich unkompliziert, aber auch „unmissverständlich".

Hans Kolaska war 41 Jahre bei der Krupp-Widia GmbH in Essen beschäftigt - davon viele Jahre als Prokurist, zuständig für die Forschungsbereiche Hartmetall, Keramik, Wärmebehandlungen und Produktionstechnik. Hier begann er mit den ersten Experimenten an Hartmetallen, die zu zahlreichen Patenten führten, $u$. a. zum weltweit ersten Patent über das Mikrowellensintern von Hartmetallen. Auch war er der erste Forscher weltweit, der 1971 das Heißisostatische Pressen (HIP) für das Nachverdichten von Hartmetallen eingesetzt hat. Während seiner "Widia-Jahren" und in den Jahren danach hat Hans Kolaska über 174 nationale und internationale Publikationen verfasst und mit der Vorlesungsreihe „Pulvermetallurgie der Hartmetalle" sowie der Herausgabe des gleichlautenden Buches maßgeblich zur Verbreitung und Verständnis seines anspruchsvollen Fachgebietes beigetragen.

Ende Dezember 1994 musste er leider aus gesundheitlichen Gründen aus der "Widia" ausscheiden, um sich nach seiner Genesung bis heute für mehr als 20 weitere Jahre einer Vielzahl von fachlichen Aktivitäten und Aufgaben zu widmen. Man kann es als Koinzidenz ansehen, dass die „Widia" bereits einen Tag nach seinem Ausscheiden, also am 01.01.1995, aufgab und vom amerikanischen Konzern Cincinatti Milacron übernommen wurde. Dem Verfasser dieses Artikels ist allerdings nicht bekannt, ob zwischen diesen beiden Ereignissen auch ein kausaler Zusammenhang bestand. Es würde aber keineswegs überraschen.

Als Berater des Fachverbandes für Pulvermetallurgie (FPM) richtete er einen Teil seiner Aktivitäten auf die Förderung des Hagener Symposiums. Seit 1986 ist er auch für die begleitende Ausstellung des Symposiums verantwortlich und konnte durch seinen unermüdlichen Einsatz die Anzahl der Aussteller von anfänglich 5 auf über 60 steigern.

Darüber hinaus war er Gründer und (zwischen 1988 und 2013) Leiter des Arbeitskreises Hartmetall im FPM. Für seine außerordentlichen Verdienste um den Ausschuss für Pulvermetallurgie und sein großes Engagement bei der Ausrichtung der Hagener Symposien wurde er 1989 mit der Ehrenplakette des Ausschusses ausgezeichnet. Es folgten Ehrungen auf

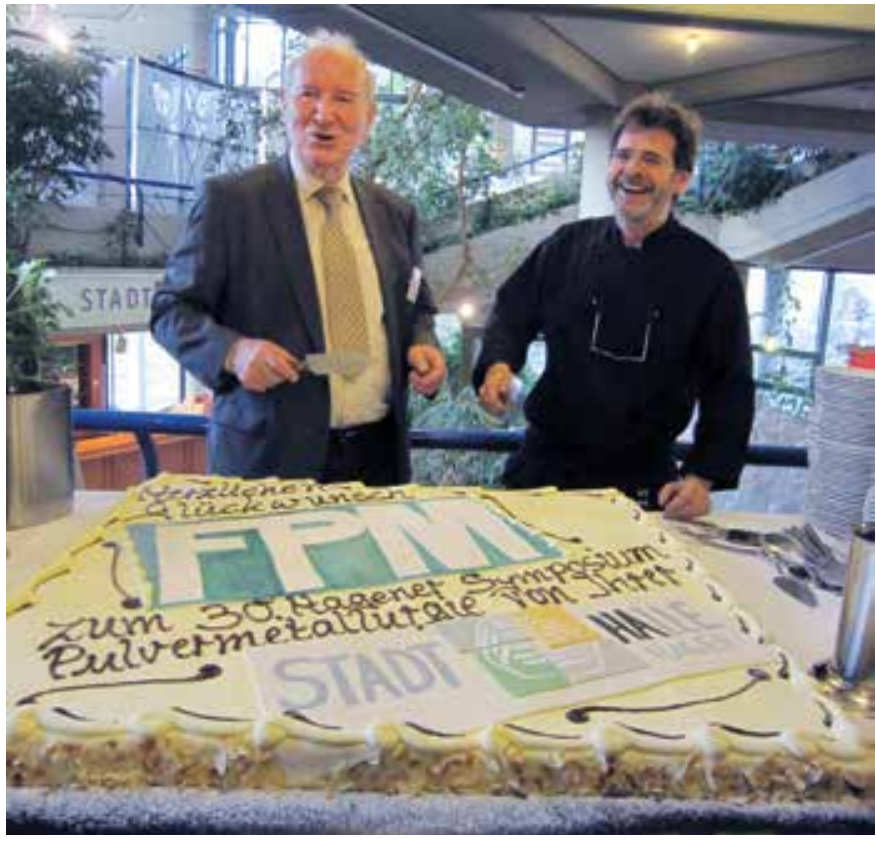

Anschnitt der Jubiläumstorte anlässlich des 30. Hagener Symposiums 2011 durch Hans Kolaska

Ehrungen, wie die Verleihung des „Sintermann 2000", der bisher nur an 4 weitere herausragende Wissenschaftler verliehen wurde. Seit 2003 ist Hans Kolaska Ehrenmitglied des "Industrieforums Werkzeuge" am Institut für Fertigungstechnik und Werkzeugmaschinen (IFW) der Universität Hannover. 2007 wurde er zum bisher einzigen Ehrenmitglied des Ausschusses für Pulvermetallurgie ernannt; 2012 erhielt er den Ehrenpreis des Instituts für Spanende Fertigung (ISF) der Universität Dortmund für 25 Jahre Förderung und Unterstützung des Instituts.

Bis heute ist Hans Kolaska sowohl Vorstandsmitglied des Fachverbandes Pulvermetallurgie (FPM) als auch der Deutschen Keramischen Gesellschaft (DKG) und Mitglied des Beirates der Messe München.

Neben seinen beruflichen bzw. fachlichen Aktivitäten hat sich der Jubilar über viele Jahre seines Lebens auch einem kleinen Ball aus Zelluloid gewidmet. In den Nachkriegsjahren erwischte ihn das Tischtennis-Fieber. 1950 schloss er sich dem TTC Bottrop 47 an. Der sportliche Ehrgeiz trug schnell Früchte: Erfolge bei den Kreis- und Bezirksmeisterschaften, Deutscher Jugendmeister im Einzel, der Aufstieg mit dem Herren-Team in die Verbandsliga (1966). Die Liste an Erfolgen, über die sich Hans Kolaska in seiner aktiven Spielerlaufbahn freuen durfte, ist umfangreich.

Dem Jubilar ist zu wünschen, dass er weiterhin viel Freude an seinen vielfältigen Aktivitäten hat - aber auch ein gesundes Maß an freier Zeit und Erholung einplant.

H. Pelc

\begin{tabular}{|c|c|}
\hline \multicolumn{2}{|c|}{$\begin{array}{l}\text { Sachverständigenbüro } \\
\text { Gernandt · Osterkamp · Stengert }\end{array}$} \\
\hline $\begin{array}{l}\text { Sachverständige für Maschinen, } \\
\text { Anlagen und Produkte der } \\
\text { Keramikindustrie } \\
\text { Loss adjusters and property } \\
\text { value assessors specialized } \\
\text { in ceramics industries }\end{array}$ & $\begin{array}{l}\text { Schadensgutachten und Wertgutachten } \\
\text { über Brand-, Produkthaftpflicht- und Gewähr- } \\
\text { leistungsschäden; über Feuerversicherungs- } \\
\text { und Verkehrswerte bei Erwerb, Beleihung } \\
\text { und fiskalischen Einschätzungen }\end{array}$ \\
\hline $\begin{array}{r}\text { D-32457 Porta W } \\
\text { Tel. +49 (0) } 5722 / 9 \\
\text { E-Mail: info@experts }\end{array}$ & $\begin{array}{l}\text { alica } \cdot \text { Kleinenbremer Straße } 16 \\
0-0 \cdot \text { Fax }+49(0) 5722 \text { / } 91290-99 \\
\text { de } \cdot \text { Internet: www.experts-gos.de }\end{array}$ \\
\hline
\end{tabular}

